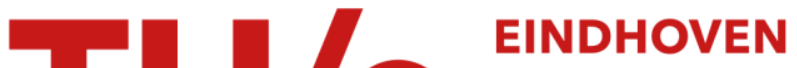 UNIVERSITY OF TECHNOLOGY
}

\section{Space syntax : standardised integration measures and some simulations}

\section{Citation for published version (APA):}

Teklenburg, J. A. F., Timmermans, H. J. P., \& Wagenberg, van, A. F. G. M. (1993). Space syntax : standardised integration measures and some simulations. Environment and Planning B: Planning and Design, 20(3), 347-357. https://doi.org/10.1068/b200347

DOI:

10.1068/b200347

Document status and date:

Published: 01/01/1993

\section{Document Version:}

Publisher's PDF, also known as Version of Record (includes final page, issue and volume numbers)

\section{Please check the document version of this publication:}

- A submitted manuscript is the version of the article upon submission and before peer-review. There can be important differences between the submitted version and the official published version of record. People interested in the research are advised to contact the author for the final version of the publication, or visit the $\mathrm{DOI}$ to the publisher's website.

- The final author version and the galley proof are versions of the publication after peer review.

- The final published version features the final layout of the paper including the volume, issue and page numbers.

Link to publication

\section{General rights}

Copyright and moral rights for the publications made accessible in the public portal are retained by the authors and/or other copyright owners and it is a condition of accessing publications that users recognise and abide by the legal requirements associated with these rights.

- Users may download and print one copy of any publication from the public portal for the purpose of private study or research.

- You may not further distribute the material or use it for any profit-making activity or commercial gain

- You may freely distribute the URL identifying the publication in the public portal.

If the publication is distributed under the terms of Article 25fa of the Dutch Copyright Act, indicated by the "Taverne" license above, please follow below link for the End User Agreement:

www.tue.nl/taverne

Take down policy

If you believe that this document breaches copyright please contact us at:

openaccess@tue.nl

providing details and we will investigate your claim. 


\title{
Space syntax: standardised integration measures and some simulations ${ }^{\dagger}$
}

\author{
J A F Teklenburg, H J P Timmermans \\ Urban Planning Group, Faculty of Architecture, Building and Planning, Eindhoven University of \\ Technology, mail station 20, PO Box 513, 5600 MB Eindhoven, The Netherlands \\ A $F$ van Wagenberg \\ Environmental Design Group, Faculty of Philosophy and Social Sciences, Eindhoven University of \\ Technology, PO Box 513,5600 MB Eindhoven, The Netherlands \\ Received 3 April 1992; in revised form 31 August 1992
}

\begin{abstract}
Central to the space syntax method (a technique for morphological analysis of urban areas) is the concept of integration. Unfortunately, the integration values are not independent of the size of urban areas. Consequently it is difficult to compare areas of different size, implying a need for standardisation of integration measures.

In this paper three such standardised integration measures are discussed. The properties of these measures are simulated for differently sized urban areas, with the assumption of a spatial structure with morphological constants. The results of these simulations suggest that a measure based on a comparison with an 'axial grid' has most desirable properties. Comments regarding the interpretation and use of the chosen standardised integration measure are provided.
\end{abstract}

\section{Introduction}

Space syntax, developed at the Unit for Architectural Studies, University College London (Hillier, 1988; Hillier and Hanson, 1984; Hiller et al, 1983; 1989a; $1989 \mathrm{~b} ; 1990)$, is a technique that can be used for morphological analysis of buildings, architectural plans, urban areas, and urban plans. The aim behind the technique is to describe different aspects of the relationships between the morphological structure of man-made environments and social structures or events.

Space syntax has been used in a wide range of research projects. Hillier et al (1987a) made an analysis of house genotypes. Peponis et al (1990) looked at the function of the morphological structure of buildings in the way-finding process. Hanson (1989) described the sociocultural implications of different plans for the rebuilding of London after the great fire. Miller (1989) used space syntax as a tool in the process of urban renewal in a Swedish town. Hillier et al (1989b) attempted to predict spatial patterns of crime in urban areas, and De Holanda (1989) was concerned with social implications of different ways of structuring cityform in the Third World. Mills (1989) showed how the spatial structure of townships acts as a mechanism of control in the apartheid ideology. Last and most important, the relationship between the morphological structure of urban areas and (mostly pedestrian) movement patterns has been researched frequently (Hillier, 1988; Hillier and Hanson, 1984; Hillier et al, 1983; 1987b; 1989a; 1990; Peponis et al, 1989).

The central concept of space syntax is integration'. It is supposed that the distribution of integration across an urban area correlates with the movement pattern in that area. Urban areas can be distinguished by, and compared in terms of,

$\dagger$ This project is financially supported by a grant from the Dutch Organisation for Scientific Research (NWO/SRO). This paper is an extended version of a paper presented at the twenty second Annual Conference of the Environmental Design Research Association, 12-15 March 1991, Oaxtepec, Mexico. 
different levels of integration. Integration is used as a measure of quality for urban areas.

The technique allows one to express integration in numeric values. In common with many other measures of spatial structure, these values are dependent upon the size of the urban area. Moreover, the distribution across the area may be biased due to the effect of the edges. These properties may not be very problematic when the aim of the research is to examine the relationship between morphology and movement pattern in a single area. However, if one wishes to test this relationship across urban areas, as in our study, these biases may constitute a serious methodological problem. Strictly speaking one is unable to conclude whether variations in integration values simply reflect size biases or represent real differences between morphological structures. In our research we try to solve the problem of bias due to the edges by relating mathematically the values of the integration measure to other features of the urban area. This can be done only when integration values do not suffer from size biases.

For these reasons a standardisation of integration measures is needed. The aim in this project is to develop an integration measure that is independent of the size of urban areas and to compare the performance of this new measure with that of existing measures of integration.

For readers less familiar with space syntax, we will first introduce the basic concepts of this technique (section 2), which will allow readers to understand the main conclusions of this paper. In section 3 we discuss the different standardised integration measures. The properties of these measures are simulated for differently sized urban areas, with the assumption of a spatial structure with morphological constants (section 4). In the final section of this paper, we discuss the results of the simulation experiments.

\section{Space syntax}

To apply space syntax it is necessary to construct a graphical representation of an urban area. This is typically done by dividing the total space of the area into public and nonpublic space. Public space can only be continuous and is called the

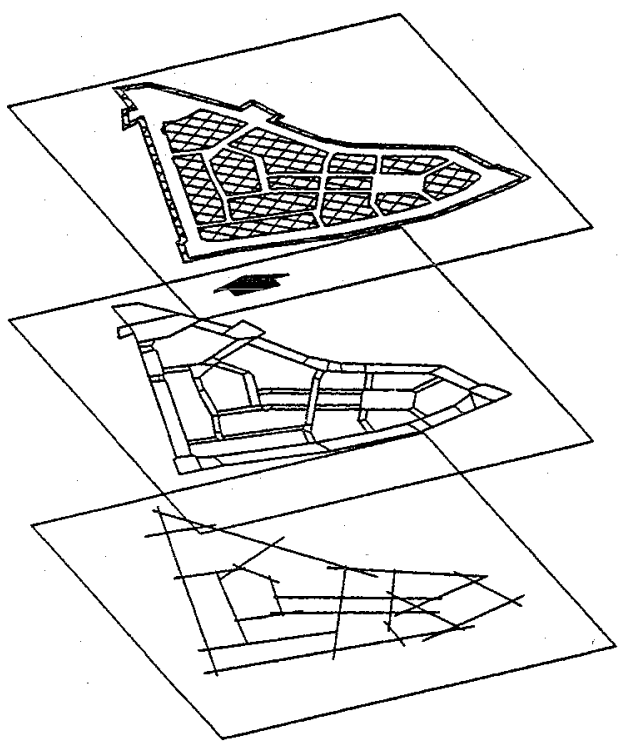

Figure 1. Open space, convex map, and axial map of an urban area.

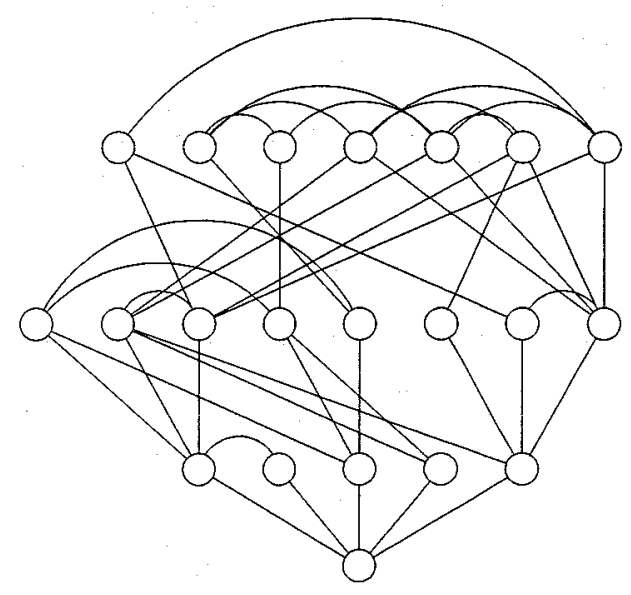

Figure 2. Graph of an axial map. 
open space of the area. This open space is divided in the least set of fattest convex spaces. By definition no tangent can be drawn on the perimeter of a convex space that passes through that space at any point.

On the basis of both the open space and the configuration of the convex spaces the axial map of an area can be drawn. The axial map contains the least set of the longest straight lines that can be drawn in the open space so that every convex space is crossed by at least one such line. The number of lines is called $L$. Figure 1 shows the open space, the convex map, and the axial map of a small neighbourhood in the city of Eindhoven, The Netherlands.

The axial map can be represented as a graph: the nodes of the graph represent the lines of the axial map; the links represent the intersections of the lines. The number of nodes corresponds to the number of lines and is also called $L$. Figure 2 shows one way to represent the axial map as a graph. The only difference between the graph and axial map is the different representation of the same features of an urban area. The axial map resembles the ground plan of an urban area but is not very convenient for computations. The graph, on the other hand, does not look like a ground plan at all, but makes computations easy. Whenever we are referring to the axial map we use 'number of lines'; referring to the graph we use 'number of nodes'.

\subsection{The concept of depth}

In space syntax, distance always means topological distance and we will refer to it as 'depth' or $D$. Depth is measured in steps. The depth between two lines that intersect is 1 . In every other case it is the minimum number of lines that must be crossed to get from one line to the other, plus 1 . The sum of the depths of a line to all the other lines of the axial map is called the total depth or ${ }_{\mathrm{T}} D$ of that line. The ${ }_{\mathrm{T}} D$ values tend to get very large and are not easy to work with. Therefore, space syntax works with the mean depth or $\bar{D}$ of lines. The mean depth of a line is defined as:

$$
\bar{D}=\frac{{ }_{\mathrm{T}} D}{L-1} \text {. }
$$

\subsection{Integration}

When a line of an axial map has a low ${ }_{\mathrm{T}} D$ or $\bar{D}$ value the axial map will look shallow from this point of view; all the other lines are very near. The axial map will look much deeper from lines with higher ${ }_{\mathrm{T}} D$ values. Maps with a low mean ${ }_{\mathrm{T}} D$ value will, from most lines, look more shallow than maps with a high mean ${ }_{\mathrm{T}} D$ value.

The distinction between deep and shallow is central to space syntax. Urban areas are described and compared in terms of the depth and shallowness of their axial maps. The distribution of these properties across the lines of the axial map is a second way to distinguish urban areas.

It must be understood that integration always means integration towards something. Axial maps with a low mean ${ }_{\mathrm{T}} D$ value are integrated towards themselves. Lines with a low $\bar{D}$ are integrated towards the other lines of the axial map. The integration of a line towards all the other lines of the axial map is calied global integration and can be expressed in the $\bar{D}$ value of the line. The integration of an axial map towards itself is called the mean global integration of the map and can be expressed as the mean of $\bar{D}$ values of the lines of the map.

The values of ${ }_{\mathrm{T}} D$ and $\bar{D}$ depend on the number of lines $(L)$ of an axial map. They increase when $L$ increases. In order to be able to compare axial maps of different size, standardisation of integration measures is needed. 


\section{Standardised integration measures}

In 1984 Hillier and Hanson introduced the measure of relative asymmetry (RA) to express integration. RA compares the deepness of an axial map from a particular line with the deepness and shallowness it theoretically could have. For any line the lowest value for ${ }_{\mathrm{T}} D$ equals $L-1$, that is when it intersects with all the other lines of the axial map. In this case $\bar{D}$ equals 1 [from equation (1)]. The highest value for ${ }_{\mathrm{T}} D$ occurs when the axial map from a particular line looks like a unilinear sequence away from that line- every additional line of the map adds one more level of depth. Thus

$$
{ }_{\mathrm{T}} D^{\max }=\sum_{n=1}^{L-1} n .
$$

As the sum of the sequence $1,2,3 \ldots(n-2),(n-1)$ is known, equation (2) can be substituted by

$$
{ }_{\mathrm{T}} D^{\max }=\frac{L(L-1)}{2} .
$$

Substitution of equation (3) in equation (1) gives

$$
\bar{D}^{\max }=\frac{L}{2} \text {. }
$$

By definition the RA of any line $(A)$ is the ratio of the distance of the $\bar{D}$ value of the line to the minimum $\bar{D}$ value $\left(\bar{D}^{\text {min }}=1\right)$ and the distance of the maximum $\bar{D}$ value $\left(\bar{D}^{\max }=\frac{1}{2} L\right)$ to the minimum value (see figure 3 ). Thus

$$
A=\frac{2(\bar{D}-1)}{L-2} \text {. }
$$

The advantage of $A$ over ${ }_{\mathrm{T}} D$ or $\bar{D}$ is that it expresses integration as a number between 0 and 1 , so it is easy to work with. Low numbers indicate high integration and vice versa.

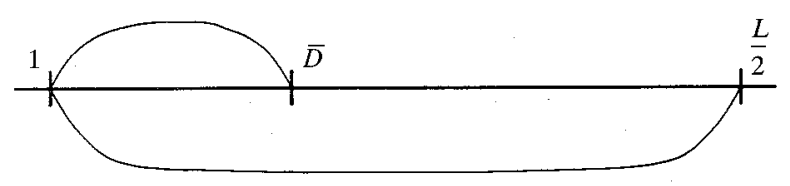

Figure 3. Construction of $A$.

\subsection{Construction of RRA}

A second measure introduced by Hillier and Hanson in their 1984 book is the real relative asymmetry (RRA), that is $A$ standardised for $L$. The RRA of a line of an axial map with $L$ lines is defined as the ratio of $A$ and the RA of a standardised line of a standardised axial map with $L$ lines. This RA is called $D_{L}$. The graph of the standardised axial map looks from the standardised line like a diamond: there are $n$ nodes at the middle level, $\frac{1}{2} n$ nodes at one level above and below the middie level, $\frac{1}{4} n$ nodes at the levels above and below the $\frac{1}{2} n$ level, $\frac{1}{8} n$ nodes at the levels above and below the $\frac{1}{4} n$ level, and so on until there is one node at the deepest level and one node (the standardised line) at the root of the graph. Figure 4 shows such a 'diamond root' with twenty-two nodes. 
Krüger (1989) argues that the distribution of the nodes across the levels is approximately normal. He shows that $D_{L}$ can be estimated by

$$
D_{L}=2\left\{L\left[\log _{2}\left(\frac{L+2}{3}\right)-1\right]+1\right\} /(L-1)(L-2) \text {. }
$$

The real relative asymmetry of a line is defined as $A^{\mathrm{D}}$ :

$$
A^{\mathrm{D}}=\frac{A}{D_{L}} \text {. }
$$

Values of $A^{\mathrm{D}}$ are larger than 0 , low values indicate strong integration, high values show weak integration.

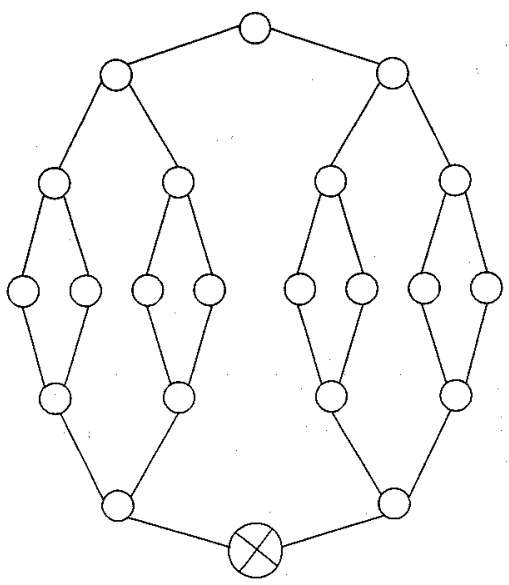

Figure 4. Diamond root graph.

\subsection{Construction of $A A^{\mathrm{G}}$,}

Krüger (1989) suggests a second way of standardising RA values. This measure is also called real relative asymmetry, but we will refer to it as ' $A$ G'.

Krüger defines the $A^{\mathrm{G}}$ of a line of an axial map with $L$ lines as the ratio of $A$ and the RA of a standardised line of a standardised axial map with $L$ lines. This RA is called $G_{L}$. The graph of the standardised axial map looks from the standardised line like a grid: there are $n$ nodes at the middle level, $n-1$ nodes at one level above and below the middle level, $n-2$ nodes at the levels above and below the $n-1$ level, $n-3$ nodes at the levels above and below the $n-2$ level, and so on until there is one node at the deepest level and one node (the standardised line) at the root of the graph. Figure 5 shows such a 'corner-of-a-grid' graph with sixteen nodes.

Krüger stipulates that the distribution of the nodes across the levels is approximately normal. He shows that $G_{L}$ can be estimated by:

$$
G_{L}=2 \frac{L(L)^{1 / 2}-2 L+1}{(L-1)(L-2)} .
$$

The $A^{\mathrm{G}}$ of Krüger is defined as:

$$
A^{\mathrm{G}}=\frac{A}{G_{L}} .
$$

The values of $A^{\mathrm{G}}$ are larger than 0 , low values indicate strong integration, high values show weak integration. 


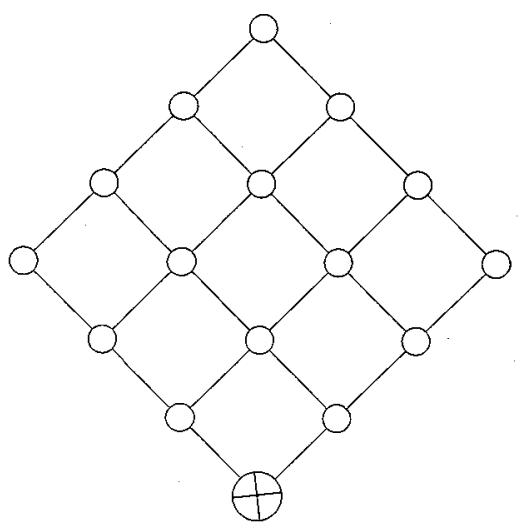

Figure 5. Corner-of-a-grid graph.

\subsection{Construction of ' $I$ '}

Both $A^{\mathrm{D}}$ and $A^{\mathrm{G}}$ are based on $A$. However, $A$ does not give any more information than ${ }_{\mathrm{T}} D$ or $\bar{D} \mathrm{do}$; namely the distribution of integration across the axial map. We constructed a standardised measure based directly on ${ }_{\mathrm{T}} D$. We call this measure the integration score (or $I$ ).

The integration score is based on the comparison of the ${ }_{\mathrm{T}} D$ of a line of an axial map with $L$ lines with the mean ${ }_{\mathrm{T}} D$ of a standardised axial map with $L$ lines.

This standardised axial map is a so-called 'axial grid': the graph of the axial map is a complete bipartite graph. Figure 6 shows an axial grid with eleven lines and its (bipartite) graph.

The properties of a complete bipartite graph are independent of the number of nodes in the graph. For that reason it is called a graph with morphological constants. The properties are as follows.

(a) The number of nodes can be any positive integer larger than 1 .

(b) When the number of nodes is even, the nodes are divided in two equally sized subsets. Every node in each subset has $\frac{1}{2} L$ links.

(c) When there are an odd number of nodes, the nodes are divided in two subsets. The first subset contains $\frac{1}{2}(L-1)$ nodes, each node in the subset having $\frac{1}{2}(L-1)+1$ links. The second subset contains $\frac{1}{2}(L-1)+1$ nodes, each node in the subset having $\frac{1}{2}(L-1)$ links.

(d) Nodes do not have links with nodes that are in the same subset. We choose the axial grid for the following reasons:

(a) An axial grid is a structure with morphological constants.

(b) An axial grid is, independent of $L$, very integrated. There can be no $D$ values between lines larger than 2 . In fact, for every line of an axial grid $\bar{D} \leqslant 1.5$.

(c) It is almost impossible to imagine real-life axial maps that will be more integrated than the axial grid. In fact, one can consider an axial grid as a real-life integration maximum.

(d) It would be very difficult to compare any axial map with the theoretical integration maximum. This would be the case when every line of the map intersects with every other line. In this situation there are only two known points: $(L-1)$ and ${ }_{\mathrm{T}} D$. We want to compare two distances (as is the case in the construction of $A$, compare with figure 3 ) and this demands at least three points.

(e) Computations based on an axial grid are simple.

The integration score of a line of an axial map with $L$ lines is defined as the ratio of the distance of the mean ${ }_{\mathrm{T}} D$ of an axial grid of $L$ lines to $L-1(L-1=$ the lowest 
${ }_{\mathrm{T}} D$ value possible for that number of lines) and the distance of ${ }_{\mathrm{T}} D$ to $L-1$ (see figure 7).

As the mean ${ }_{\mathrm{T}} D$ of an axial grid with an even number of lines equals the ${ }_{\mathrm{T}} D$ of any line of the grid, it can be easily calculated. There are $\frac{1}{2} L$ nodes in every subset of the graph. Every node has a depth of 1 to the nodes in the subset to which it does not belong (equal to $\frac{1}{2} L$ nodes). It has a depth of 2 to the nodes in the subset it belongs to (equal to $\frac{1}{2} L-1$; nodes do not have depth to themselves). Thus, for an axial grid with an even number of lines,

$$
{ }_{\mathrm{T}} D^{\text {mean }}=\frac{3 L-4}{2} \text {. }
$$

The situation for an axial grid with an uneven number of lines is somewhat different. A node in the subset $\frac{1}{2}(L-1)$ has a depth of 1 to the nodes in the subset $\frac{1}{2}(L-1)+1$ and a depth of 2 to the $\frac{1}{2}(L-1)-1$ nodes of the subset it belongs to. Thus

$$
{ }_{\mathrm{T}} D=\frac{3 L-5}{2} \text {. }
$$

A node in the subset $\frac{1}{2}(L-1)+1$ has a depth of 1 to the node in the subset $\frac{1}{2}(L-1)$, and a depth of 2 to the $\frac{1}{2}(L-1)$ nodes of the subset it belongs to. Thus

$$
{ }_{\mathrm{T}} D=\frac{3 L-3}{2} \text {. }
$$

Combining equations (11) and (12) leads to the mean $D_{\mathrm{T}}$ of an axial grid with an odd number of lines:

$$
{ }_{\mathrm{T}} D^{\text {mean }}=\frac{L(3 L-4)+1}{2 L} .
$$
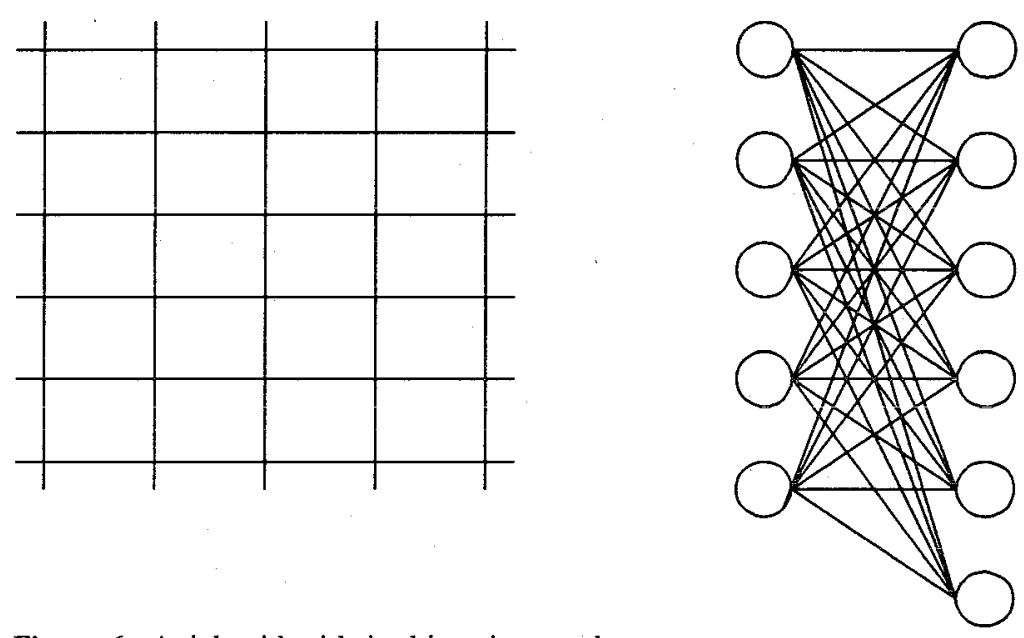

Figure 6. Axial grid with its bipartite graph.

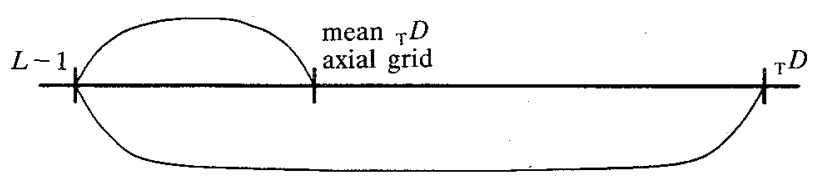

Figure 7. Construction of $I$. 
Mean $A^{\mathrm{D}}, A^{\mathrm{G}}$, and $I$ values were computed for graphs with $3 \leqslant L \leqslant 703$ (703 being the maximum number of nodes our computer could handle for this type of graph).

\subsection{Results of the simulation experiments}

The results of the computations are shown in figure 10. The diagrams clearly show that mean $A^{\mathrm{D}}$ values increase when $L$ increases, except for the complete bipartite graph, in which case they decrease when $L$ increases. The conclusion must be that mean $A^{\mathrm{D}}$ values are in all cases highly dependent on $L$.

Mean $A^{\mathrm{G}}$ values decrease when $L$ increases in the case of the axial grid, they increase with increasing $L$ for maximal deep axial maps. In these cases the mean $A^{\mathrm{G}}$ values are highly dependent on $L$. This is not so for axial maps with a regular or triangular node grid graph: mean $A^{\mathrm{G}}$ values then seem to be independent of $L$.

In all four cases mean $I$ values are independent of $L$.

\section{Summary}

The goal of this paper was to decide what kind of standardisation of the integration measure would make its values independent of the number of lines of the axial map of an urban area. There were two reasons why this is important. Independence makes it possible to compare urban areas of different size, and it makes computations based on the integration measure reliable in the sense that its values are less influenced by operational decisions regarding the construction of the axial map and especially the delimitation of the study area.

Our simulations do not prove that for all axial maps mean $I$ values are less dependent of the number of lines than mean $A^{\mathrm{D}}$ or mean $A^{\mathrm{G}}$ values. But it is clear that even for more extreme axial maps with morphological constants (unilinear sequence and complete bipartite graph) the mean values of $I$ are stable. For axial maps with regular or triangular grid node graphs the stability of mean $A^{\mathrm{G}}$ values is satisfying. However, this measure cannot cope with the more extreme axial maps.

These simulations do not indicate the stability of individual $I$ values. However, the stability of individual $I$ values is of only minor importance. The mean $I$ values allow the comparison of urban areas of different size. In any given urban area the distribution of $I$ values corresponds perfectly with the distribution of integration. As the range of individual $I$ values in all four simulation experiments is very small (less than 0.6), and is in fact much smaller than for $A^{\mathrm{D}}$ (greater than 45) or $A^{\mathrm{G}}$ (greater than 10), $I$ values can be considered to be a reliable basis for computations.

Equation (14) is meaningless for $L \leqslant 2$ and for ${ }_{\mathrm{T}} D \leqslant L$. This means that an average $I$ cannot be calculated for an axial map containing a line with ${ }_{\mathrm{T}} D \leqslant L$. Although this is very unlikely to happen in any urban area, one can imagine an architectural situation that satisfies this condition. Therefore, it is advisable to use the integration score primarily for urban plans or very large buildings. The distribution of integration can of course still be calculated by using ${ }_{\mathrm{T}} D$ values, if an average $I$ cannot be calculated.

\section{References}

De Holanda F, 1989, "Brasilia: the daily invention of the city" Ekistics 56 (334/335) 75-83

Hanson J, 1989, "Order and structure in urban design: the plans for the rebuilding of London after the Great Fire of 1666" Ekistics 56 (334/335) 22-42

Hillier B, 1988, "Against enclosure", in Rehumanising Housing Eds N Teymur, T Markus, T Wooley (Buttersworths, London) pp 63-85

Hillier B, Hanson J, 1984 The Social Logic of Space (Cambridge University Press, Cambridge)

Hillier B, Hanson J, Graham H, 1987a, "Ideas are in things: an application of the space

syntax method to discovering house genotypes" Environment and Planning B $14363-385$ 
Hillier B, Hanson J, Penn A, 1989a, "Natural movement: or, configuration and attraction in urban pedestrian movement", paper for the European Conference on the Representation and Management of Urban Change, Cambridge, 28-29 September; copy available from Unit for Architectural Studies, University College London

Hillier B, Burdett R, Peponis J, Penn A, 1987b, "Creating live: or, does architecture determine anything?" Architecture and Behaviour $3233-250$

Hillier B, Grajewski T, Jianming X, Jones L, 1989b The Spatial Pattern of Crime on the Studley Estate Unit for Architectural Studies, University College, London

Hillier B, Grajewski T, Jones L, Jianming X, Greene M, 1990 Broadgate Spaces: Life in Public Spaces Unit for Architectural Studies, University College, London

Hillier B, Hanson J, Peponis J, Hudson J, Burdett R, 1983, "Space syntax: a new urban perspective" Architects Journal 178 (48) 48-63

Krüger M J T, 1989, "On node and axial grid maps, distance measures and related topics", paper for the European Conference on the Representation and Management of Urban Change, Cambridge, 28-29 September, Unit for Architectural Studies, University College London

Miller J, 1989, "Growth and renewal: the Swedish model" Ekistics 56 (334/335) 56-64

Mills G, 1989, "Space and power in South Africa: the township as a mechanism of control" Ekistics $56(334 / 335) 65-74$

Peponis J, Hadjinikolaou E, Livieratos C, Fatouros D A, 1989, "The spatial core of urban culture" Ekistics 56 (334/335) 43-55

Peponis J, Zimring C, Choi Y K, 1990, "Finding the building in wayfinding" Environment and Behavior $22555-590$ 
